STRUCTURAL SCIENCE CRYSTAL ENGINEERING MATERIALS

ISSN 2052-5206

Keywords: crystal engineering; metalloligand; praseodymium salt; MOF; supramolecular compound; iodine adsorption.

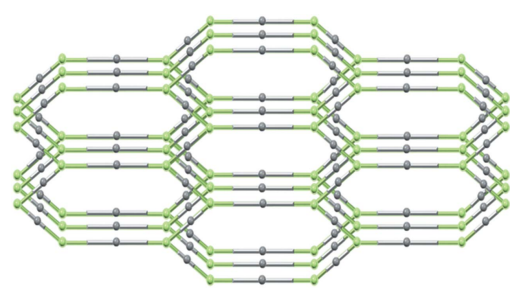

C 2020 International Union of Crystallography

\section{In and out: crystal engineering for reversible iodine uptake}

\author{
Guillermo Minguez Espallargas*
}

Instituto de Ciencia Molecular (ICMol), Universidad de Valencia, c/ Catedrático José Beltrán, 2, Paterna, 46980, Spain. *Correspondence e-mail: guillermo.minguez@uv.es

In this issue of Acta Crystallographica Section B, Moghzi et al. (2020) report the chemical design of a metal-organic compound with an outstanding iodine $\left(\mathrm{I}_{2}\right)$ sorption capability. Sequestration of $\mathrm{I}_{2}$, an extremely volatile gas, has important applications in terms of controlling radioactive gases, as various radionuclides of $\mathrm{I}_{2}$ are released during the fission of nuclear fuels. Thus, the preparation of porous materials that can efficiently capture and store $I_{2}$ is of importance not only from an academic point of view, but also for real-life applications.

In their report, the authors nicely illustrate the process that has been followed to prepare the final material, demonstrating the importance of careful evaluation of the different crystal structures obtained. In other words, Moghzi and co-workers show an example of the crystal engineering process, i.e. the understanding of intermolecular interactions in the context of crystal packing and the utilization of such understanding in the design of new solids with desired physical and chemical properties. In this sense, they have fine-tuned the synthetic conditions in order to control the dimensionality of the product, from discrete units (0D), to chains (1D) and layers (2D). More specifically, the authors show that the dimensionality of the final coordination polymer is dependent on the coordination mode of the $\mathrm{N}$-heterocyclic polycarboxylic acid used (pyridine-2,6dicarboxylic acid or pyrazine-2,3-dicarboxylic acid). This, in turn, depends on the $\mathrm{pH}$ of the initial solution, which is varied with the addition of piperazine, a base that also acts a second ligand. These findings, key for the successful chemical design, have been made possible thanks to a detailed analysis of the crystal structures.

In order to further expand the dimensionality of the coordination polymers to form a 3D metal-organic framework (or MOF), Moghzi and co-workers replaced the $\mathrm{N}$ heterocyclic polycarboxylic acid for a $\mathrm{Zn}$-based metalloligand, with the aim of connecting the 2D layers in the third direction thus forming a 3D structure. The combination of the metalloligand with a praseodymium salt results in a bimetallic crystalline material which expands in the three dimensions, as was anticipated by the authors. In addition, this structure is thermally stable (up to $400^{\circ} \mathrm{C}$ ), an important aspect for applications.

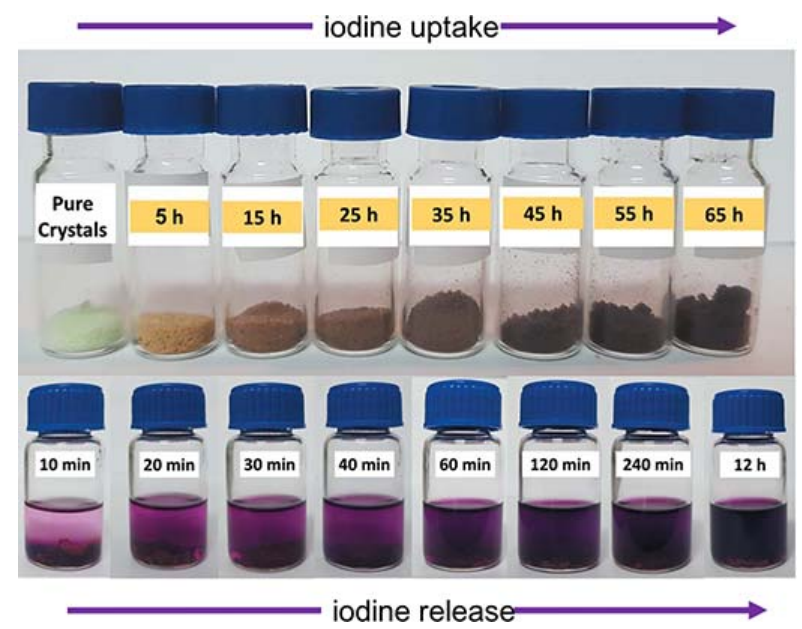

Figure 1

Combined photographic images taken from two figures in paper by Moghzi et al. (2020) showing the colour change on iodine uptake and iodine release. 


\section{scientific commentaries}

This MOF, upon exposure to iodine vapors, is capable of incorporating $\mathrm{I}_{2}$ molecules, as revealed by the gradual color change of the solid, which turns dark brown over time (see Fig. 1, top). In addition, gravimetric measurements reveal a mass increase over time, with a remarkable maximum amount of sorbed $\mathrm{I}_{2}\left(490 \mathrm{mg} \mathrm{g}^{-1}\right)$ after $45 \mathrm{~h}$. This value places this novel material among the best MOFs in terms of $\mathrm{I}_{2}$ sorption.

The reverse process, i.e. the release of $\mathrm{I}_{2}$, has also been investigated by this team. In particular, they used cyclohexane, a common organic solvent, to extract the incorporated $\mathrm{I}_{2}$, and used spectroscopic techniques to follow the process. However, as iodine solutions are strongly colored, the process can also be followed visually, as can be seen in Fig. 1 (bottom). Still,
UV-vis spectroscopy was used to confirm that release is completed after $4 \mathrm{~h}$.

Once the reversibility of the process was established, Moghzi and co-workers studied its cyclability, revealing that the capacity of this compound remains almost intact after four cycles of adsorption-desorption. Although clearly this is not a massive number of cycles, it is a first step that reveals the potential of the material for iodine sequestration.

\section{References}

Moghzi, F., Soleimannejad, J., Emadi, H. \& Janczak, J. (2020). Acta Cryst. B76, 779-788. 